\title{
DETERMINANTES DE \\ LOS INGRESOS LABORALES DE \\ LOS ECONOMISTAS EN COLOMBIA UN ANÁLISIS DE MODELACIÓN MICRO ECONOMÉTRICA
}

León J. Arango Buelvas

Universidad de Sucre

Colombia

Gustavo A. González Palomino Universidad de Sucre Colombia

César A. Escobar Vergara Universidad de Sucre Colombia

Shadia R. Paternina Vergara Universidad de Sucre Colombia 
Panorama Económico, Vol. 27 - No. 1 (Enero - Marzo de 2019), pp. 85-104

León J. Arango Buelvas

Gustavo A. González Palomino

César A. Escobar Vergara

Shadia R. Paternina Vergara

JEL: C30, J40, J44,

\title{
Determinantes de los ingresos laborales de los economistas en Colombia: Un análisis de modelación micro econométrica
}

\begin{abstract}
Resumen
En la presente investigación se analizan los factores determinantes de los ingresos laborales de los economistas en Colombia para el tercer trimestre de los años 2010 y 2016, en el marco de una perspectiva teórica de capital humano. Con información de la Gran Encuesta Integrada de Hogares del Departamento Administrativo Nacional de Estadística (DANE), se estima una ecuación minceriana clásica para medir el efecto de la escolaridad y la experiencia potencial en los ingresos de los titulados en economía. Después de aplicar el método de Mínimos Cuadrados Ordinarios, los resultados sugieren que un año adicional de formación escolar y entrenamiento laboral en el puesto de trabajo genera un efecto positivo sobre la probabilidad de devengar ingresos laborales más altos. Adicionalmente, aplicando el modelo de Mincer para las profesiones afines se pudo evidenciar que las variables de capital humano tienen impactos significativamente diferentes para cada grupo de profesionales, por lo cual la hipótesis planteada de un mercado laboral homogéneo no se cumple para el periodo de estudio.
\end{abstract}

Palabras clave: Ingreso laboral, ecuación de Mincer, capital humano, escolaridad, experiencia.

\section{Déterminants du revenu du travail pour les économistes en Colombie: une analyse de modélisation micro économétrique}

\begin{abstract}
Résumé
Cette recherche analyse les déterminants du revenu du travail des économistes en Colombie pour le troisième trimestre 2010 et 2016, dans le cadre d'une perspective théorique du capital humain. D'après les informations de la grande enquête intégrée auprès des ménages du Département administratif national des statistiques (DANE), une équation minérérienne classique est estimée pour mesurer l'effet de la scolarité et l'expérience potentielle sur les revenus des diplômés en économie. Après avoir appliqué la méthode des moindres carrés ordinaires, les résultats suggèrent qu'une année supplémentaire de scolarité et de formation en cours d'emploi a un effet positif sur la probabilité de gagner un revenu d'emploi plus élevé. De plus, en appliquant le modèle de Mincer pour les professions apparentées, il était évident que les variables du capital humain ont des impacts significativement différents pour chaque groupe de professionnels, par conséquent l'hypothèse d'un marché du travail homogène n'est pas remplie pour la période d'étude.
\end{abstract}

Mots clés: Revenu du travail, équation de Mincer, capital humain, scolarité, expérience.

\section{Determinants of Labor Income for Economists in Colombia: A micro econometric modeling analysis}

\begin{abstract}
This research analyzes the determinants of labor income of economists in Colombia for the third quarter of 2010 and 2016, within the framework of a theoretical perspective of human capital. With information from the Large Integrated Household Survey of the National Administrative Department of Statistics (DANE), a classical mincerian equation is estimated to measure the effect of schooling and potential experience on the incomes of graduates in economics. After applying the Ordinary Least Squares method, the results suggest that an additional year of school education and on-the-job job training has a positive effect on the probability of earning higher job earnings. Additionally, applying the Mincer model for related professions, it was evident that the human capital variables have significantly different impacts for each group of professionals, therefore the hypothesis of a homogeneous labor market is not fulfilled for the study period.
\end{abstract}

Keywords: Labor Income, Mincer equation, human capital, schooling, experience. 


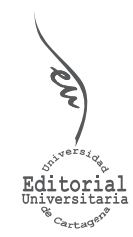

Artículo de Investigación

\section{Determinantes de los ingresos laborales de los economistas en Colombia: Un análisis de modelación micro econométrica}

INFORMACIÓN DEL ARTíCULO

Recepción del artículo: 03/07/2018

Concepto de evaluación: 18/08/2018

Aceptación del artículo: 05/10/2018
León J. Arango Buelvas* Universidad de Sucre, Colombia

Gustavo A. González Palomino** Universidad de Sucre, Colombia

César A. Escobar Vergara Universidad de Sucre, Colombia

Shadia R. Paternina Vergara Universidad de Sucre, Colombia

\section{INTRODUCCIÓN}

La formación académica y personal durante el periodo escolar, así como el entrenamiento laboral comprenden decisiones de inversión que se evalúan bajo un panorama de incertidumbre sobre consideraciones futuras (Martínez Gutiérrez, 2012). Este tipo de decisiones implican un análisis de costo-beneficio y una evaluación de los rendimientos futuros esperados de la productividad del trabajo durante el ciclo de vida laboral. A partir de los trabajos pioneros de Becker (1962), Schultz (1963), Ben Porath (1967) y Mincer (1974), la literatura económica de los mercados de trabajo ha estudiado ampliamente los determinantes de los ingresos laborales, demostrando que la acumulación de conocimientos y habilidades son factores determinantes en la obtención de beneficios futuros pecuniarios y no pecuniarios para los individuos.

Sin embargo, Phelps (1972), Arrow (1973), Spence (1973) y Stiglitz (1978) consideraron que los cambios en los niveles de productividad de un trabajador no sólo dependen de sus propias características individuales de capital, sino también de factores externos a las condiciones particulares de los individuos que explican los cambios en los niveles de productividad laboral (El-Attar \& López-Bazo, 2009). Según Piña (2006), la complementariedad entre capital humano y educación puede manifestarse no solamente en la mayor productividad resultado de la capacitación sino de la movilización y utilización más intensa del capital entre profesionales.

\footnotetext{
*Profesor Asociado del Programa de Economía de la Universidad de Sucre. ORCID: 0000-0001-8198-1872. Autor de correspondencia: leon.arango@unisucre.edu.co

**Universidad de Sucre. ORCID: 0000-0002-0698-1714. Contacto: gustavo.gonzalez@unisucre.edu.co
} 
De acuerdo con los informes estadísticos de demanda laboral en Colombia, las áreas de conocimientos como Economía, Administración de Empresas y Contaduría Pública tienen un porcentaje de vinculación laboral de aproximadamente 79,7\% del total nacional (DANE, 2015a). Estos resultados reflejan un mercado laboral con altas posibilidades de vinculación para los titulados en economía, aunque también con mayor competencia dentro del mismo (DANE, 2015b). Este escenario hace relevante el estudio propuesto, ya que constituye una base fundamental para comprender los elementos que posibilitan el acceso a mejores ingresos laborales; sobre ello la presente investigación se orienta a responder el siguiente interrogante: ¿Cuáles son los determinantes de los ingresos laborales de los economistas en Colombia para el tercer trimestre de los años 2010 y 2016?

En este sentido, se analiza el efecto de los rendimientos de la escolaridad y la experiencia potencial en las remuneraciones percibidas por los titulados en Economía. En consonancia con el desarrollo del objetivo principal se tendrán en consideración tres objetivos específicos; el primero de ellos busca caracterizar sociodemográficamente el mercado laboral de los economistas en Colombia para los años 2010 y 2016; el segundo se orienta a describir los elementos que definen la remuneración salarial de estos profesionales para el mismo periodo de estudio, con lo cual se posibilitará el tercer objetivo que permitirá comparar los principales elementos explicativos del ingreso laboral de los economistas frente a las profesiones afines como Administración de Empresas y Contaduría Pública.

Para ello, se toman como referencia los datos de la Gran Encuesta Integrada de Hogares (GEIH) del Departamento Administrativo Nacional de Estadística (DANE) para el tercer trimestre de los años 2010 y 2016. Posteriormente se estima un modelo empírico de ecuaciones mincerianas incluyendo variables de capital humano como: Escolaridad, Experiencia potencial y Experiencia al cuadrado.

La estructura del presente artículo inicialmente aborda un apartado teórico y revisa la literatura referente a los factores determinantes de los ingresos laborales a nivel internacional y nacional. Seguidamente, se revela el enfoque metodológico y su operacionalización, luego se analizan las estadísticas descriptivas del mercado laboral de los economistas en Colombia y se discuten los ejercicios econométricos de los modelos mincerianos estimados. Finalmente, se exponen las conclusiones obtenidas en la investigación y se presentan las referencias bibliográficas.

\section{REVISIÓN DE LITERATURA}

Existe un creciente interés de la literatura especializada por estimar los determinantes salariales de los agentes económicos en el mercado laboral, para ello se acude al uso de ecuaciones mincerianas y funciones generatrices de ingresos, las cuales regularmente contemplan fuentes de información representadas en ejercicios econométricos que tienen en cuenta la educación, la experiencia laboral y atributos relacionados con el trabajador, para ofrecer una visión del mercado de trabajo.

En este acervo empírico, a nivel internacional se encuentra el estudio de Almendarez y Urciaga (2008), los cuales analizan los ingresos laborales en la frontera norte de México, a partir de los rendimientos privados de la educación, indicando que los retornos de la escolaridad guardan relación directa y permanente con el salario. Teniendo en cuenta la información estadistica consignada en la Encuesta Nacional de Empleo Urbano 2002 (ENEU), estiman el siguiente modelo minceriano: 


$$
L n_{W H O R A}=\beta_{0}+\beta_{1} E D U+\beta_{2} E X P E R A+\beta_{3} E X P E R A^{2}+\mu
$$

Donde, Ln_WHORA es el logaritmo natural de los ingresos por hora; EDU es el número de años de educación formal recibida; EXPERA son los años de experiencia laboral potencial; EXPERA ${ }^{2}$ es el cuadrado de la experiencia potencial; y $\mu$ es el término de perturbación aleatoria. Dentro de los hallazgos obtenidos, encuentran que el retorno de la educación formal es de aproximadamente el 10\%, asociado a factores que tienen en cuenta el nivel de estudios y la renta salarial, que se incrementa con los años de educación. Así mismo, la conducta del vínculo de la edad y su cuadrado con los ingresos sigue una tendencia en forma de U invertida, alcanzando percepciones máximas a los 45 años.

Por otro lado, Sapelli (2003) plantea un análisis exploratorio de los determinantes del salario y las tasas de retorno de la educación en Chile durante los años 1990 y 1998, con datos transversales de la Encuesta de Caracterización Socioeconómica Nacional (CASEN). Para ello, acude a la ecuación original de Mincer:

$$
\beta_{0}+\beta_{1} S+\beta_{2} X+\beta_{3} X^{2}++\mu
$$

Respondiendo al logaritmo del ingreso real (ln_((y) )) a partir de los años de escolaridad (S), los años de experiencia laboral potencial $(\mathrm{X})$ y la experiencia potencial al cuadrado $\left(\mathrm{X}^{\wedge} 2\right)$ del encuestado. Con los resultados obtenidos, concluye que existe relación significativa entre los ingresos laborales de los ocupados y los años de escolaridad que posee cada individuo, señalando que el retorno promedio del componente educativo es de $11,4 \%$ para el año 1990 y 13,2\% para 1998 en las remuneraciones salariales percibidas por los intergrupos de asalariados.

En el ámbito nacional, Tarazona y Remolina (2017) siguiendo una ecuación tipo Mincer, calculan el efecto en las remuneraciones salariales de la tasa de retorno de la educación y la experiencia en Colombia para los años 2009 y 2016, a partir de los microdatos extraídos de la Gran Encuesta Integrada de Hogares (GEIH) del Departamento Administrativo Nacional de Estadística (DANE), incluyendo como variables explicativas el nivel de escolarización (Edu), la experiencia laboral potencial (Exp), la experiencia al cuadrado (Exp²) y el término de error estadístico, así:

$$
\ln _{(y)}=\beta_{0}+\beta_{1} S+\beta_{2} X+\beta_{3} X^{2}++\mu \quad[3]
$$

Dentro de los resultados encontrados, resaltan que la tasa de retorno de la educación para el territorio nacional es de 9,1\%, mientras que un año más de experiencia podría aumentar los ingresos de un individuo al menos en $2,2 \%$. Si el individuo acumula muchos años de experiencia, un año adicional podría disminuir sus ingresos en 0,002\%. 


\section{MARCO TEÓRICO}

Existe amplia diversidad literaria que ha analizado y cuantificado los determinantes de los ingresos de los trabajadores en el mercado laboral. La teoría del capital humano, propuesta por Mincer (1958), Schultz (1961) y Becker (1962), se ha convertido en uno de los principales referentes para comprender los determinantes de las remuneraciones salariales, dando evidencia empírica que resalta la importancia de la inversión en capital humano como factor causal de los ingresos laborales de aquellos individuos que toman decisiones de invertir en educación (Cortés \& Flórez, 2016).

Este enfoque en el mercado laboral sostiene que los ingresos laborales de los individuos, se explican por diferencias en su capital humano, desde la óptica del stock educativo o la experiencia que se adquiere en el puesto de trabajo (Becker, 1964). En el marco del planteamiento anterior, Jacob Mincer (1974) desarrolla una función que permite estudiar los efectos de la inversión en años de educación formal recibida y los años de experiencia laboral en los ingresos laborales de los individuos, atentos a su ciclo de vida, denotándose como sigue:

$$
\ln W i=\beta_{0}+\beta_{1} S_{i}+\beta_{2} X_{i}+\beta_{3} X_{i}^{2}+\varepsilon_{i}
$$

Donde $\mathrm{W}$ son los ingresos del individuo; $\mathrm{S}$ es el número de años de educación formal recibida; $\mathrm{X}$ son sus años de experiencia laboral potencial ${ }^{1}$; la cual se calcula teniendo en cuenta la modificación de los años trabajados por un individuo que labora tiempo completo cuando finaliza sus estudios y $\varepsilon$ es el término de perturbación aleatoria.

Los postulados que afirmaban que la educación garantizaba a los individuos mayores incrementos en la productividad se replantean cuando Arrow (1973), Spence (1973) y Stiglitz (1975) introducen el concepto de señalización, basados en la hipótesis de la utilidad de la educación como un mecanismo a través del cual los individuos adquieren títulos que emplean como señales para que los empleadores establezcan expectativas acerca de su productividad (Iglesias Garrido, 2005a). La señalización implica un proceso de autoselección, en el cual los individuos pueden crear sus posibilidades de salarios y de puestos de trabajo al educarse, porque a través de la enseñanza adquieren las señales que los posicionan de manera diferente ante el mercado de trabajo. Desde el punto de vista de los trabajadores, desde que perciben la posibilidad de aumentar las ganancias a través de la información de sus propias habilidades, intentarán aumentar los recursos destinados a adquirir esta información (Iglesias Garrido, 2005b).

Según lo mencionado anteriormente, la educación y la experiencia podrían ser los principales elementos explicativos de la determinación de los salarios, lo que se recoge en los planteamientos de la teoría del capital humano y la señalización. No obstante, el presente estudio se sustenta en el fundamento teórico propuesto por Becker (1962), quién modifica la teoría neoclásica en un supuesto fundamental: el factor trabajo no es homogéneo sino diferenciado en categorías ocupacionales y otros factores de calidad de la mano de obra.

1. Marchionni (2001) propone un ajuste metodológico a la experiencia laboral potencial de la siguiente forma: $\mathrm{X}=$ a-S-6; donde la experiencia laboral potencial (X) se especifica como la cantidad de años (a) que potencialmente un individuo puede haber trabajado, menos los años de educación formal (S) y menos 6, la edad a la cual empieza su proceso de educación. 
Becker (1962) introduce el concepto del capital humano como el principal elemento explicativo de la heterogeneidad del trabajo y de las diferencias salariales; los individuos acuden al mercado de trabajo con niveles diferentes de stocks de capital humano de acuerdo con su dotación innata y la formación que poseen, originando la diversidad de grupos o subgrupos que no son fácilmente sustituibles entre sí. Las heterogeneidades de capital humano en el corto plazo, provocan diferencias salariales debido a las discrepancias productivas entre trabajadores. A largo plazo, los individuos pueden trasladarse a ocupaciones mejor remuneradas, pero la intensidad de movimiento se determina por las inversiones en capital humano y la capacidad inherente de los individuos para asimilar la educación y la formación (McConnell, Brue, \& MacPherson, 2007).

\section{METODOLOGÍA}

El presente artículo sigue un diseño deductivo con enfoque descriptivo y cuantitativo, ya que pretende identificar los factores determinantes del ingreso laboral de los economistas en Colombia para el tercer trimestre de los años 2010 y 2016. En primer lugar, se requerirá de información secundaria, obtenida del Departamento Administrativo Nacional de Estadística (DANE) a partir de los microdatos con corte transversal de la Gran Encuesta Integrada de Hogares (GEIH) para el tercer trimestre de los años 2010 y 2016 . El tamaño de la muestra que siguen los microdatos corresponde a 80.079 individuos al año 2010 y 74.387 para el año 2016, teniendo cobertura geográfica para áreas urbanas (cabecera), áreas rurales (resto) y áreas metropolitanas. Para el desarrollo de la investigación se hace una depuración de los capítulos: 10 (Características generales), 50 (Fuerza de trabajo), 60 (Ocupados) ${ }^{2}, 70$ (Desocupados) por medio del Software STATA/SE 12.0.

Para estudiar los efectos de la inversión en educación y formación laboral en el nivel, composición y distribución interpersonal de los ingresos en el ciclo de vida de los individuos, así como determinar si existe heterogeneidad de dotaciones en capital humano entre cada grupo de profesionales o si la disparidad de los ingresos laborales no puede ser explicada por diferencias en productividades individuales, se emplea la función de ingresos de Mincer bajo Mínimos Cuadrados Ordinarios (MCO) para economistas (oficio09), contadores (oficio11), administradores de empresas (oficio21) y Colombia (Col), planteándose de la siguiente forma semilogarítmica:

$$
\begin{gathered}
\ln W_{\text {oficio09 }}=\beta_{0}+\beta_{1} S_{i}+\beta_{2} X_{i}+\beta_{3} X_{i}^{2}+\varepsilon_{i} \\
\ln W_{\text {oficio } 11}=\beta_{0}+\beta_{1} S_{i}+\beta_{2} X_{i}+\beta_{3} X_{i}^{2}+\varepsilon_{i} \\
\ln W_{\text {oficio } 21}=\beta_{0}+\beta_{1} S_{i}+\beta_{2} X_{i}+\beta_{3} X_{i}^{2}+\varepsilon_{i} \\
\ln W_{\text {Col }}=\beta_{0}+\beta_{1} S_{i}+\beta_{2} X_{i}+\beta_{3} X_{i}^{2}+\varepsilon_{i}
\end{gathered}
$$

\footnotetext{
2. La pregunta que permite clasificar la ocupación de la población ocupada en el formulario de la Gran Encuesta
} Integrada de Hogares (GEIH) se encuentra en el capítulo 60, codificada así: ¿Qué hace en este trabajo? (OFICIO). 
Donde, (ln W) es el logaritmo del ingreso (en el caso específico de esta investigación se tomó como variable dependiente el logaritmo natural del salario de economistas, contadores, administradores y Colombia); las variables independientes son los años de educación $\left(\mathrm{S}_{\mathrm{i}}\right)$; la experiencia laboral $\left(\mathrm{X}_{\mathrm{i}}\right)$; el cuadrado de la experiencia $\left(\mathrm{X}_{\mathrm{i}}{ }^{2}\right)$ y el término de error de estimación ( $\left.\varepsilon^{\mathrm{i}}\right)$, con la particularidad de corresponder a cada oficio ${ }^{3}$.

Si se supone que se desarrollan los supuestos en los que opera la función minceriana, el valor del coeficiente de los años de educación formal $B_{1}$ ) debe ser positivo y constante al arrojar un estimador de la tasa de retorno de un año adicional de estudio adquirido por los individuos. Se espera que al ser la función cóncava con respecto a la experiencia, se obtengan estimaciones de $\mathrm{B}_{2}$ positivas y de $\mathrm{B}_{3}$ negativas, indicando que las decisiones de los individuos por tener mayor nivel de experiencia, generan mayor productividad laboral esperada y, por tanto mejores ingresos salariales (Freire \& Mercedes, 2010).

En la aplicación empírica de este tipo de modelos mincerianos, suele evidenciarse una serie de errores de sesgo en la estimación de parámetros debido a la dificultad en la medición de ciertas variables. Los estimativos de los coeficientes tienden a sesgarse por la exclusión de los individuos que no laboran al tiempo que se realiza la medición (Tenjo, Rivero, \& Bernat, 2005). Por tanto, se procede a realizar las respectivas correcciones a través de la metodología definida por Heckman (1979), mediante la cual se desarrollan estimaciones en dos etapas: una inicial, en la que se estima un modelo probit que calcula la probabilidad de los individuos pertenecientes a la población en edad de trabajar (PET) de hacer parte del mercado de trabajo. Luego de estimarse la ecuación probabilística, se hace el cálculo del inverso de la razón de Mill ( $\Lambda$ ) y finalmente, se estima un modelo (MCO), introduciendo el valor de lambda ( $\Lambda$ ) como regresor adicional en la ecuación (8) de ingresos. Debe aclararse que al no disponer de frecuencia censurada por reporte de ingreso laboral a nivel de oficios, no se puede estimar la ecuación de corrección de selectividad muestral para los economistas. Para el caso de Colombia, los resultados obtenidos mediante la modelación bietápica de Heckman, mantuvieron resultados consistentes.

Por otra parte, con el objetivo de determinar si la dotación innata de capital humano es un elemento explicativo en los ingresos laborales de los economistas en el mercado laboral colombiano, se modifica la ecuación original de Mincer, de la siguiente manera:

$$
\ln W i=\beta_{0}+\beta_{1} S_{i}+\beta_{2} X_{i}+\beta_{3} X_{i}^{2}+\beta_{4} \text { oficio0 } 9 n_{i}+\mu_{i}
$$

Siendo, (ln Wi) el logaritmo natural de los ingresos laborales de la fuerza de trabajo en Colombia, explicados por los años de educación $\left(\mathrm{S}_{\mathrm{i}}\right)$, la experiencia laboral potencial $\left(\mathrm{X}_{\mathrm{i}}\right)$, el cuadrado de la experiencia potencial $\left(\mathrm{X}_{\mathrm{i}}^{2}\right)$, el atributo de desempeñarse como economista ( $\mathrm{B}_{4}$ oficio 09 $\mathrm{n}_{\mathrm{i}}$ ) y el término de error de estimación $\left(\mathrm{u}_{\mathrm{i}}\right)$.

Seguidamente, para verificar la presencia de sesgo de selección muestral en la ecuación (9) y obtener estimadores consistentes y asintóticamente eficientes bajo el supuesto de normalidad y homocedasticidad del término de error no censurado, se acude a la metodología definida por Heckman (1979), usando la siguiente ecuación de participación laboral: 


$$
P E A=\beta_{0}+\beta_{1} p a r+\beta_{2} e c+\beta_{3} e d+\varepsilon_{i}
$$

Donde PEA es la población económicamente activa; par es una variable dicotómica que alude al parentesco, registrando valor de 1 sí es jefe de hogar, 0 en otro caso; ec es la variable dicotómica -estado conyugal- que toma el valor de 1 si el encuestado es casado, 0 en otro caso y ed alude a la edad del mismo.

\section{RESULTADOS}

\section{Caracterización del mercado laboral de los economistas en Colombia}

Este apartado contiene un análisis descriptivo de indicadores demográficos, laborales y educativos para el mercado laboral de los economistas en Colombia, correspondiente al tercer trimestre de los años 2010 y 2016. De igual forma, se presenta una distinción comparativa de variables de capital humano entre los profesionales en Economía, con respecto a la media nacional y a profesiones afines como Contaduría Pública y Administración de Empresas.

Tabla 1.

Estadísticas descriptivas del mercado laboral de los economistas y profesiones afines en Colombia para los años 2010 y 2016.

\begin{tabular}{|c|c|c|c|c|c|c|c|c|}
\hline & \multicolumn{2}{|c|}{ Economistas } & \multicolumn{2}{|c|}{ Contadores } & \multicolumn{2}{|c|}{ Administradores } & \multicolumn{2}{|c|}{ Colombia } \\
\hline & \multicolumn{8}{|c|}{2010} \\
\hline Variables & Media & Std. Err. & Media & Std. Err. & Media & Std. Err. & Media & Std. Err. \\
\hline Escolaridad & 16.09 & 2.46 & 16.71 & 1.71 & 15.98 & 3.07 & 5.25 & 4.45 \\
\hline Exp. potencial & 18.34 & 15.54 & 17.78 & 9.95 & 22.73 & 13.18 & 15.59 & 22.06 \\
\hline Edad & 39.7 & 16.24 & 39.4 & 9.95 & 41.2 & 11.88 & 29.8 & 20.68 \\
\hline Ingreso & $\$ 1.995 .178$ & $\$ 1.215 .325$ & $\$ 2.230 .860$ & $\$ 2.847 .488$ & $\$ 2.385 .114$ & $\$ 3.974 .069$ & $\$ 712.548$ & $\$ 1.546 .699$ \\
\hline Observaciones & \multicolumn{2}{|c|}{73} & \multicolumn{2}{|c|}{683} & \multicolumn{2}{|c|}{1742} & \multicolumn{2}{|c|}{80.079} \\
\hline \multirow[t]{2}{*}{$\begin{array}{l}\text { Obs con factor } \\
\text { de expansión }\end{array}$} & \multicolumn{2}{|c|}{21.214} & \multicolumn{2}{|c|}{122.158} & \multicolumn{2}{|c|}{400.230} & \multicolumn{2}{|c|}{17.827 .901} \\
\hline & \multicolumn{8}{|c|}{2016} \\
\hline Variables & Media & Std. Err. & Media & Std. Err. & Media & Std. Err. & Media & Std. Err. \\
\hline Escolaridad & 16.64 & 1.27 & 16.74 & 1.6 & 14.43 & 3.82 & 5.59 & 4.64 \\
\hline Exp. potencial & 13.55 & 8.77 & 16.2 & 11.07 & 21.84 & 12.09 & 16.75 & 23.18 \\
\hline Edad & 35.2 & 8.86 & 37.94 & 11.12 & 41.28 & 11.24 & 35.48 & 10.75 \\
\hline Ingreso & $\$ 3.228 .612$ & $\$ 2.401 .256$ & $\$ 2.457 .236$ & $\$ 2.378 .271$ & $\$ 3.614 .463$ & $\$ 5.276 .593$ & $\$ 672.453$ & $\$ 1.276 .395$ \\
\hline Observaciones & \multicolumn{2}{|c|}{31} & & & & & & \\
\hline $\begin{array}{l}\text { Obs con factor } \\
\text { de expansión }\end{array}$ & \multicolumn{2}{|c|}{11.088} & & & & & & \\
\hline
\end{tabular}

Fuente: DANE, Gran Encuesta Integrada de Hogares (2010 y 2016). Elaboración Propia 
En la tabla I se evidencia tendencia creciente en la variación de la escolaridad media para el caso de los economistas, contadores y Colombia, exceptuando a administradores de empresas los cuales redujeron el promedio de los años de educación en un 9,7\% del año 2010 al 2016. Cabe anotar que para el caso de los graduados en Economía se refleja crecimiento nominal en dicha variable al tiempo que cae el nivel de dispersión, por lo que se intuye que la educación media creció en un valor representativo en el mercado laboral. Por otro lado, la experiencia potencial media disminuyó ligeramente entre las profesiones mencionadas, aunque, para el mercado laboral colombiano, se evidencia crecimiento nominal del parámetro medio que describe esta variable.

Un elemento a resaltar dentro del análisis del mercado laboral de los profesionales en Economía en Colombia, tiene que ver con el hecho de que el ingreso laboral ${ }^{4}$ presentó tendencia creciente a lo largo de los años estudiados, reportando incremento del $61,82 \%$, reflejado en la transición de $\$ 1.995 .178$ pesos colombianos mensuales (2010), a \$ 3.228.612 pesos colombianos mensuales en 2016, con diferencial positivo entre los años de alrededor \$1.233.434. Sin embargo, el ingreso laboral medio de los economistas para el año 2010, estuvo por debajo de profesiones afines como Contaduría Pública y Administración de Empresas. No obstante, para el tercer trimestre del año 2016, el ingreso promedio de los economistas, superó el reporte salarial de los contadores y la media nacional, pero se mantuvo ligeramente por debajo de los administradores de empresas.

Cabe resaltar que para ambos periodos, la media del ingreso laboral de los graduados en Economía se mantuvo por encima de su desviación estándar, denotando mayor homogeneidad y representatividad en el mercado laboral que las profesiones afines, que se caracterizaron por registrar mayor nivel de dispersión en las remuneraciones salariales.

\section{Ejercicio econométrico}

Con el objetivo de encontrar los factores determinantes del ingreso laboral de los economistas en Colombia, se realizó la estimación de una ecuación minceriana que permite estudiar los efectos de la inversión en años de educación formal recibida y los años de experiencia laboral en los ingresos laborales de la población estudiada, a partir de la información de la Gran Encuesta Integrada de Hogares (GEIH) para los años 2010 y 2016. En la tabla II, se exponen los resultados de la ecuación de Mincer por el método de Mínimos Cuadrados Ordinarios (MCO) y Máxima Verosimilitud (MV). Es de aclarar, que a todas las regresiones se les aplicó supuesto de corrección de White (1980) con la intención de eliminar la heterocedasticidad presente en los datos de corte transversal. 
Tabla 2

Estimaciones de ecuaciones mincerianas para economistas y carreras afines en Colombia 2010 y 2016.

\begin{tabular}{|c|c|c|c|c|}
\hline \multirow[t]{2}{*}{ Año } & \multicolumn{4}{|c|}{2010} \\
\hline & \multicolumn{2}{|c|}{ Economistas } & \multicolumn{2}{|c|}{ Contadores } \\
\hline \multirow{2}{*}{$\begin{array}{c}\text { MÉTODO } \\
\text { VARIABLES }\end{array}$} & \multicolumn{2}{|c|}{ M.C.O } & \multicolumn{2}{|c|}{ M.C.O } \\
\hline & COEF. & STD. ERR & COEF. & STD. ERR \\
\hline Escolaridad & $0.0954454^{* * * *}$ & $(0.0201135)$ & $0.1188442^{* * * *}$ & $(0.0175868)$ \\
\hline Exp. potencial & $0.0630519^{* * * *}$ & $(0.0195403)$ & $0.0270573^{* * * *}$ & (0.0076081) \\
\hline $\operatorname{Exp}^{2}$ & $-0.001130^{* * * *}$ & $(0.0003901)$ & $-0.000412^{* * * *}$ & $(0.0001816)$ \\
\hline \multicolumn{5}{|l|}{ Parentesco } \\
\hline \multicolumn{5}{|l|}{ Estado conyugal } \\
\hline \multicolumn{5}{|l|}{ Edad } \\
\hline Intercepto & $12.10323^{* * * *}$ & $(0.3823522)$ & $11.91874^{* * * *}$ & $(0.2868783)$ \\
\hline \multicolumn{5}{|l|}{ RHO } \\
\hline \multicolumn{5}{|l|}{ SIGMA } \\
\hline \multicolumn{5}{|l|}{ LAMBDA } \\
\hline F statistic & $10.93^{* * * *}$ & & $25.27^{* * * *}$ & \\
\hline $\mathrm{R}^{2}$ & 0.3034 & & 0.1080 & \\
\hline \multicolumn{5}{|l|}{ Wald Test (CHI2) } \\
\hline Observaciones & 73 & & 679 & \\
\hline \multicolumn{5}{|l|}{ Obs. Censuradas } \\
\hline \multicolumn{5}{|l|}{ Lr Test $(\mathrm{RHO}=0)$} \\
\hline Año & \multicolumn{4}{|c|}{2016} \\
\hline Escolaridad & $0.0601445^{* * * *}$ & $(0.0653376)$ & $0.1074657^{* * * *}$ & \\
\hline Exp. potencial & $0.0383094^{* * * *}$ & $(0.0385503)$ & $0.0399602^{* * * *}$ & \\
\hline Exp & $-0.0004699^{* * * *}$ & $(0.0009484)$ & $-0.0009484^{* * * *}$ & \\
\hline \multicolumn{5}{|l|}{ Parentesco } \\
\hline \multicolumn{5}{|l|}{ Estado conyugal } \\
\hline \multicolumn{5}{|l|}{ Edad } \\
\hline Intercepto & $13.16729^{* * * *}$ & (1.00419) & $12.20922^{* * * *}$ & $(0.3660459)$ \\
\hline \multicolumn{5}{|l|}{ RHO } \\
\hline \multicolumn{5}{|l|}{ SIGMA } \\
\hline \multicolumn{5}{|l|}{ LAMBDA } \\
\hline F statistic & $3.21^{*}$ & & $17.18^{* * * *}$ & \\
\hline $\mathrm{R}^{2}$ & 0.1803 & & 0.0975 & \\
\hline \multicolumn{5}{|l|}{ Wald Test (CHI2) } \\
\hline Observaciones & 30 & & 445 & \\
\hline \multicolumn{5}{|l|}{ Obs. Censuradas } \\
\hline \multicolumn{5}{|l|}{ Lr Test $(\mathrm{RHO}=0)$} \\
\hline$* * *=a 0,01$ & $*=a 0,05$ & 10 & & \\
\hline
\end{tabular}


Tabla 2.

\section{Estimaciones de ecuaciones mincerianas para economistas y carreras afines en Colombia 2010 y 2016.}

\begin{tabular}{|c|c|c|c|c|c|c|}
\hline \multirow[t]{2}{*}{ Año } & \multicolumn{6}{|c|}{2010} \\
\hline & \multicolumn{3}{|c|}{ Administradores } & \multicolumn{3}{|c|}{ Colombia } \\
\hline \multirow{2}{*}{$\begin{array}{l}\text { MÉTODO } \\
\text { VARIABLES }\end{array}$} & \multicolumn{2}{|c|}{ M.C.O } & \multicolumn{2}{|c|}{ M.C.O } & \multicolumn{2}{|c|}{ M.V } \\
\hline & COEF. | & STD. ERR & COEF. & STD. ERR & COEF. | & STD. ERR \\
\hline Escolaridad & $0.1285413^{* * * *}$ & $(0.0059394)$ & $0.110658^{* * * *}$ & (0.07698) & $0.103851^{* * * *}$ & (0.0007388) \\
\hline Exp. potencial & $0.0306362^{* * * *}$ & $(0.0057817)$ & $0.040485^{* * * *}$ & $(0.0007242)$ & $0.023583^{* * * *}$ & $(0.0006888)$ \\
\hline $\operatorname{Exp}^{2}$ & $-0.000211^{* *}$ & $(0.0001135)$ & $-0.00051^{* * * *}$ & $(0.0000124)$ & $-0.00033^{* * * *}$ & $(0.0000116)$ \\
\hline Parentesco & & & & & $0.664112^{* * * *}$ & $(0.0086273)$ \\
\hline Estado conyugal & & & & & $0.185316^{* * * *}$ & $(0.0067034)$ \\
\hline Edad & & & & & $0.0011873^{* * * *}$ & $(0.0002028)$ \\
\hline Intercepto & $11.78315^{* * * *}$ & $(0.1106163)$ & $11.4619^{* * * *}$ & (0.0133577) & $12.5538^{* * * *}$ & $(0.0161369)$ \\
\hline RHO & & & & & -0.871836 & $(0.003987)$ \\
\hline SIGMA & & & & & 1.11418 & $(0.0061354)$ \\
\hline LAMBDA & & & & & -0.971383 & $(0.0091646)$ \\
\hline F statistic & $172.27^{* * * *}$ & & $8288.33^{* * * *}$ & & & \\
\hline $\mathrm{R}^{2}$ & 0.2592 & & 0.2496 & & & \\
\hline Wald Test (CHI2) & & & & & $26301.83^{* * * *}$ & \\
\hline Observaciones & 1742 & & 78.739 & & 168.581 & \\
\hline Obs. Censuradas & & & & & 89.842 & \\
\hline Lr Test $(\mathrm{RHO}=0)$ & & & & & $6507.69^{* * * *}$ & \\
\hline
\end{tabular}

\begin{tabular}{|c|c|c|c|c|c|c|}
\hline \multirow{2}{*}{$\frac{\text { Año }}{\text { Escolaridad }}$} & \multicolumn{6}{|c|}{2016} \\
\hline & $0.1192701^{* * * *}$ & $(0.0074843)$ & $0.10018^{* * * *}$ & $(0.0010275)$ & $0.095751^{* * * *}$ & (0.0009125) \\
\hline Exp. potencial & $0.0394674^{* * * *}$ & $(0.004817)$ & $0.03311^{* * * *}$ & $(0.0008853)$ & $0.020319^{* * * *}$ & $(0.0008076)$ \\
\hline Exp & $-0.000524^{* * * *}$ & $(0.0000943)$ & $-0.00046^{* * * *}$ & (0.0000155) & $-0.00030^{* * *}$ & $(0.0000141)$ \\
\hline Parentesco & & & & & $0.391683^{* * * *}$ & $(0.0067515)$ \\
\hline Estado conyugal & & & & & $0.120202^{* * * *}$ & (0.0064318) \\
\hline Edad & & & & & $0.000541^{* * * *}$ & (0.0001818) \\
\hline Intercepto & $12.1081^{* * * *}$ & $(0.1257944)$ & $11.9445^{* * * *}$ & $(0.0170964)$ & $13.4203^{* * * *}$ & $(0.019004)$ \\
\hline RHO & & & & & -0.930823 & $(0.0022175)$ \\
\hline SIGMA & & & & & 1.276154 & (0.009088) \\
\hline LAMBDA & & & & & -1.187873 & (0.0106571) \\
\hline F statistic & $127.44^{* * * *}$ & & $4274.42^{* * * *}$ & & & \\
\hline $\mathrm{R}^{2}$ & 0.2565 & & 0.2287 & & & \\
\hline Wald Test (CHI2) & & & & & $15598.07^{* * * *}$ & \\
\hline Observaciones & 1209 & & 51.499 & & 155.055 & \\
\hline Obs. Censuradas & & & & & 103.556 & \\
\hline Lr Test $(\mathrm{RHO}=0)$ & & & & & $10052.15^{* * * *}$ & \\
\hline
\end{tabular}

${ }^{* * * *}=\mathrm{a} 0,01 \quad{ }^{* * *}=\mathrm{a} 0,05 \quad{ }^{* *}=\mathrm{a} 0,10$

Fuente: DANE, Gran Encuesta Integrada de Hogares (2010 y 2016). Elaboración Propia 
Una primera aproximación empírica revela que los resultados obtenidos, a través de la ecuación minceriana clásica tienen consistencia teórica con los signos de las tasas de retornos de las variables descritas en la teoría de Mincer. Dado el caso de que los ingresos laborales están expresados funcionalmente en logaritmo natural y hay regresores en escala numérica, los coeficientes deben expresarse como semielasticidades (Wooldridge, 2006).

Los coeficientes positivos de las variables escolaridad y experiencia potencial guardan relación directa con el logaritmo de los ingresos laborales, lo cual explicaría que un cambio relativo en alguna de las tasas de retorno de dichas variables, tenderá a un cambio en el mismo sentido en la variable dependiente, que en tal caso representa un aumento en la posibilidad de percibir mayores ingresos. Por otro lado, la variable experiencia potencial al cuadrado, al ser negativa guarda concordancia a nivel teórico con el supuesto que el rendimiento de los salarios comienza a decrecer a partir de cierto número de años de experiencia, asociada con los planteamientos del ciclo de vida de la teoría minceriana, describiendo que pueden darse incrementos en la capacidad productiva relacionado con la inversión en educación en el transcurso de la vida laboral pero que disminuyen en los últimos años de trabajo.

Globalmente, los modelos estimados por las distintas metodologías arrojan resultados paralelos, los signos de los coeficientes y su significancia estadística se mantienen en las diferentes regresiones con buenos niveles de ajuste: las de Mínimos Cuadrados Ordinarios (MCO) muestran buen R2, relativamente bajo por su característica de ser datos de corte transversal, mientras que las de Máxima Verosimilitud (MV) presentan Wald Test (Chi2) representativo, el cual al ser estadísticamente significativo rechaza la hipótesis nula de no significancia. El modelo en particular, describe que las tasas de retorno que más impactan el logaritmo de los ingresos laborales para los economistas en ambos años son la escolaridad y la experiencia. A partir de los resultados encontrados, es posible afirmar que para el año 2010 todas las variables incluidas en la modelación son estadísticamente significativas para explicar los ingresos laborales de los economistas en $1 \%$ de significancia, demostrando que los regresores tienen capacidad representativa en la función.

Una tasa mayor de rendimiento medio de un año adicional de educación formal para los titulados en Economía, reflejó un incremento del 9,54\% en sus ingresos laborales al año 2010. Así también, un año adicional de experiencia en el puesto de trabajo se tradujo en un aumento del 6,30\% de la remuneración salarial percibida por estos profesionales. Además, para el año 2016, una tasa mayor de rendimiento promedio de un año adicional de educación significó un aumento del 6,01\% en sus ingresos laborales. Así mismo, en cuanto al rendimiento concerniente a la experiencia, un año adicional de trabajo, representó $3,83 \%$ de incremento salarial. Se podría afirmar que estas tasas de rentabilidad difieren de las primeras en proporción pequeña, probablemente por razones estadísticas como el tamaño de la muestra o bien por el tipo de datos y la dimensión espacial.

Sin embargo, al hacer el análisis de las tasas de retorno de la educación y la experiencia potencial para las profesiones afines de Contaduría Pública y Administración de Empresas, así como Colombia en general, se pudo constatar que los coeficientes de los retornos de estas mismas variables para ambas carreras, estuvieron por encima del retorno educativo y formativo de los graduados en Economía para el tercer trimestre de los años estudiados. Esta situación refleja que podría haber otros factores no especificados en las ecuaciones mincerianas, que hacen que los años de escolaridad y la experiencia sean más explicativos para las carreras afines que para los economistas. 
Es importante resaltar que cuando se estiman ecuaciones mincerianas, podrían obtenerse coeficientes sesgados debido a la exclusión de información sobre los ingresos laborales de quienes no están participando laboralmente. Para el caso específico de los economistas y demás profesiones, es necesario advertir que no se puede hacer filtro de corrección de selectividad de Heckman, debido a que los datos estimados por Mínimos Cuadrados Ordinarios no disponen de frecuencia censurada por oficio, porque necesariamente están ligadas al empleo y este a su vez al reporte del ingreso laboral. En consecuencia, es posible estimar la rutina de ecuaciones planteadas mediante el método MCO. No obstante, para el caso de Colombia fue necesario estimar los resultados a través del modelo bietápico de Heckman, que permitió calcular mediante el método de Máxima Verosimilitud (MV), un modelo Probit con el objetivo de determinar la ecuación de selección. Posteriormente, a partir de la estimación Probit, se obtuvo el valor estimado de lambda $(\Lambda)$, el cual arrojó la probabilidad de estar en la muestra sobre la probabilidad de no estarlo.

De acuerdo con los resultados corregidos, el retorno de la escolaridad para Colombia en el año 2010 se redujo mínimamente en 0,68 puntos porcentuales, pasando de 11,06\% con sesgo a 10,38\% corregido (MV). En el caso de los rendimientos de la experiencia potencial, el retorno también disminuyó, pasando de 4,04\% en $\mathrm{MCO}$, a 2,35\% sin sesgo. El coeficiente de la experiencia al cuadrado aumentó de -0,00051 a 0,00033 en 2010. Para el año 2016, al igual que el primer año de estudio, las tasas de retorno de la escolaridad, la experiencia potencial y la experiencia al cuadrado se redujeron de forma mínima con la corrección de selección muestral; en el caso de la rentabilidad de la educación, la tasa de retorno pasó de $10,01 \%$ sesgado a $9,57 \%$ corregido, mientras que la experiencia potencial y la experiencia al cuadrado se redujeron con el filtro de selectividad en $1,28 \%$ y $0,016 \%$, respectivamente.

Los resultados guardan coherencia con las conclusiones obtenidas en investigaciones previamente realizadas en el ámbito nacional como las de Forero \& Gamboa (2007); Psacharopoulos \& Vélez (1992); Riascos H. \& Erazo (2010) y Ortiz Benavides, Erazo, \& Burgos (2015), las cuales han demostrado que tanto la educación formal como la experiencia en el puesto de trabajo, tienen impacto positivo en el incremento de la productividad marginal, compensado con mayores niveles de ingreso laboral.

\section{Ejercicio adicional}

Con el propósito de analizar el efecto diferencial "ser economista" en el logaritmo de los ingresos laborales en Colombia, se realizó la estimación de una ecuación minceriana original, incluyendo como regresor adicional el oficio correspondiente a los profesionales en Economía, descrito en el módulo de ocupados dentro de la Gran Encuesta Integrada de Hogares (GEIH), a partir de la Clasificación Nacional de Ocupaciones- SENA 70. La incorporación de esta variable dicotómica permite visualizar si además de la escolaridad y la experiencia potencial, la cualidad "ser economista" garantiza un retorno sobre el crecimiento de los ingresos laborales del mercado de trabajo en Colombia, durante los años 2010 y 2016. En la tabla III, se muestran los resultados obtenidos de las ecuaciones de Mincer ampliadas por el método de Mínimos Cuadrados Ordinarios (MCO) y Máxima Verosimilitud (MV), incluyendo la corrección por el filtro de selectividad de Heckman, eliminando la heterocedasticidad en los datos de corte transversal por supuesto de corrección de White (1980). 
Tabla 3.

\section{Estimación de ecuación minceriana con efecto diferencial para economistas en Colombia}

\begin{tabular}{|c|c|c|c|c|}
\hline \multirow{3}{*}{$\begin{array}{c}\text { MÉTODO } \\
\text { VARIABLES }\end{array}$} & \multicolumn{2}{|c|}{2010} & \multicolumn{2}{|c|}{2016} \\
\hline & \multicolumn{2}{|c|}{ M.C.O } & \multicolumn{2}{|c|}{ M.C.O } \\
\hline & COEF. & STD. ERR & COEF. & STD. ERR \\
\hline Escolaridad & $0.1104^{* * * *}$ & (0.0201135) & $0.1000^{* * * *}$ & $(0.0010)$ \\
\hline Exp. potencial & $0.0405^{* * * *}$ & $(0.0195403)$ & $0.0331^{* * * *}$ & $(0.0008)$ \\
\hline $\operatorname{Exp}^{2}$ & $-0.0005^{* * * *}$ & (0.0003901) & $-0.0004^{* * * *}$ & $(0.0000)$ \\
\hline Oficio09n & $0.4982^{* * * *}$ & & $0.6251^{* * * *}$ & $(0.0930)$ \\
\hline \multicolumn{5}{|l|}{ Parentesco } \\
\hline \multicolumn{5}{|l|}{ Estado conyugal } \\
\hline \multicolumn{5}{|l|}{ Edad } \\
\hline Intercepto & $11.462^{* * * *}$ & $(0.3823522)$ & $11.945^{* * * *}$ & $(0.0170)$ \\
\hline \multicolumn{5}{|l|}{ RHO } \\
\hline \multicolumn{5}{|l|}{ SIGMA } \\
\hline \multicolumn{5}{|l|}{ LAMBDA } \\
\hline F statistic & $6246.3^{* * * *}$ & & $3234.8^{* * * *}$ & \\
\hline $\mathrm{R}^{2}$ & 0.2499 & & 0.2289 & \\
\hline \multicolumn{5}{|l|}{ Wald Test (CHI2) } \\
\hline Observaciones & 78739 & & 51499 & \\
\hline \multicolumn{5}{|l|}{ Obs. Censuradas } \\
\hline Lr Test (RHO=0) & & & & \\
\hline
\end{tabular}

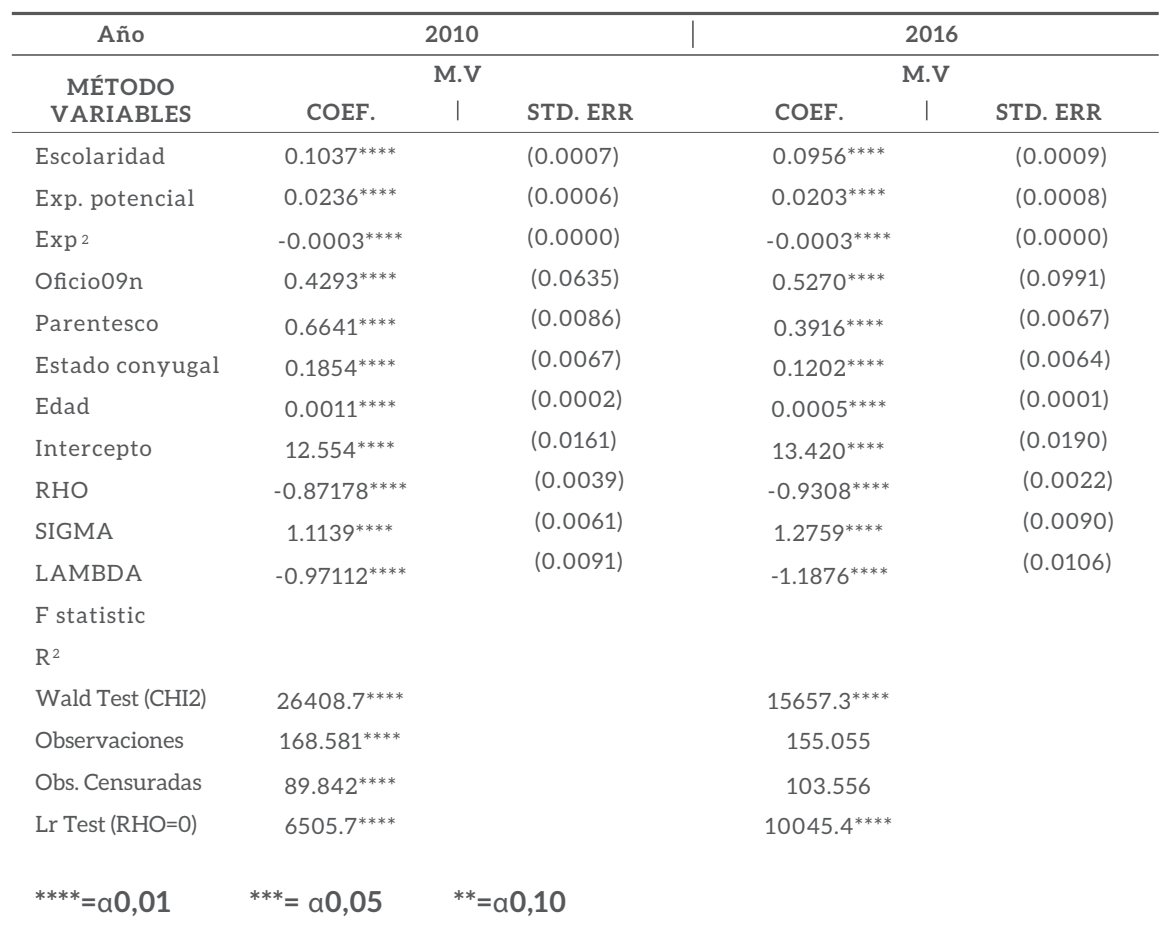

Fuente: DANE, Gran Encuesta Integrada de Hogares (2010 y 2016). Elaboración Propia 
Los resultados obtenidos, a través de las ecuaciones mincerianas ampliadas con el "efecto economista" al igual que el modelo original, guardan concordancia teórica con los signos de las tasas de rentabilidad de las variables descritas en la teoría minceriana. Es importante resaltar que los coeficientes señalados en la especificación empírica del modelo ampliado son coherentes con la perspectiva teórica de capital humano, en la medida que cuanto mayor es el stock educativo del individuo, mayor es también el premio salarial que obtienen en el mercado de trabajo (Salas, 2003). Pero, los retornos salariales caen por el efecto marginal decreciente de la experiencia.

Los coeficientes obtenidos por el método de Mínimos Cuadrados Ordinarios (MCO) y Máxima Verosimilitud (MV) a través del modelo bietápico de Heckman son estadísticamente significativos al 1\%. Luego de aplicar la corrección por filtro de selectividad, las proporciones no resultaron ser distantes con las de MCO. La bondad de ajuste de ambos modelos, puede considerarse razonablemente admisible, debido a la naturaleza y estructura de la información reportada. Por su parte, el estadístico F lleva a intuir que las variables en su conjunto son relevantes y tienen capacidad explicativa de los ingresos laborales en Colombia para los años de estudio.

El modelo corregido, describe que la cualidad "ser economista" generó un efecto diferencial positivo de 0.4293 (sin sesgo) en el crecimiento de las remuneraciones salariales en el mercado laboral colombiano para el año 2010. Así también, un año adicional de escolaridad y experiencia en el puesto de trabajo, representó aumentos de 0.1037 y 0.236 en las rentas salariales, respectivamente. Para el año 2016, el "efecto economista" aumentó en 0.2277 con respecto al 2010, generando diferencial en los ingresos laborales de 0.5270, casi 0.10 puntos porcentuales por encima del registrado en el primer año de estudio. Sin embargo, las tasas de retorno de las variables de capital humano como escolaridad y experiencia potencial cayeron 0.078 y 0.1386 , respectivamente. Estos resultados son correspondientes con estudios similares en el orden nacional, como los de Farné (2000) y Forero \& Ramírez (2008), demostrando empíricamente que los profesionales del área de ciencias económicas, administrativas y contables tienen mayor probabilidad de devengar ingresos laborales altos por su tipo de empleabilidad en el mercado de trabajo.

\section{CONCLUSIONES}

Los resultados obtenidos permiten concluir que la inversión en capital humano, a través de la escolaridad y la experiencia potencial, son determinantes en la obtención de mayores ingresos laborales de los economistas en Colombia durante el tercer trimestre de los años 2010 y 2016. Los indicadores laborales en el mercado de trabajo para el periodo de estudio, reflejan que el ingreso laboral medio de los titulados en Economía tuvo una tendencia creciente mayor (0.61) si se compara con las variaciones de los registros salariales de Administradores de Empresas (0.51) y Contadores Públicos (0.10). Cabe aclarar que el promedio salarial de los economistas se caracterizó por presentar un patrón homogéneo con bajo nivel de dispersión si se contrasta con las profesiones afines.

A través de la estimación de la ecuación de Mincer mediante el método de Mínimos Cuadrados Ordinarios (MCO), verificando su validez y superando la presencia de heterogeneidad no observada en el término de perturbación, se reafirma la hipótesis que existe relación positiva entre el ingreso salarial y la escolaridad en el mercado laboral de los economistas en Colombia para el período estudiado. Un año adicional de educación incrementa aproximadamente en 9,54\% y 6,01\% el ingreso laboral de los titulados en Economía para los años 2010 y 2016, respectivamente. Se podría afirmar que las tasas de 
rentabilidad de la educación difieren entre ellas en proporción pequeña, probablemente por razones estadísticas como el tamaño de la muestra o bien por el tipo de datos y la dimensión espacial.

Esta investigación establece, por medio de estimaciones econométricas, que los años de experiencia potencial resultan significativos, estadísticamente hablando, al momento de determinar su efecto en los ingresos laborales, pues un año adicional de experiencia en el puesto de trabajo aumenta en 6,30\% (2010) y 3,83\% (2016) las remuneraciones salariales de los graduados en esta área de conocimiento. Estos resultados son congruentes con estudios internacionales y nacionales, como el de Brenes Narváez (2005); Gómez \& Rojas (2014) y Ortiz Benavides, Erazo \& Burgos (2015).

La tasa marginal de retorno de la educación sugiere que al pasar de un grado de formación educativa a otro se incrementan los incentivos para participar en el mercado de trabajo, teniendo acceso a remuneraciones más altas. Esto indica que los ingresos laborales están directamente relacionados con las capacidades y habilidades de los individuos, concediendo un rol importante a los factores de oferta del mercado laboral. Además, los hallazgos permiten establecer que existe relación positiva entre el ingreso laboral y la experiencia potencial de los economistas, cumpliendo el supuesto establecido en la teoría de los perfiles de edad e ingresos. Se observa que, a medida que aumenta el entrenamiento en el trabajo, los perfiles alcanzan el máximo de ingreso con más años de experiencia, pero a partir de ahí el descenso de los mismos es cada vez más acentuado.

Por otra parte, al hacer la descomposición de los retornos de las variables de capital humano entre economistas, profesiones afines (Administración de Empresas - Contaduría Pública) y Colombia, se pudo establecer que existen diferencias promedio de 0.14 y 0.06 en las tasas de retorno de la escolaridad y de la experiencia potencial en los reportes de los ingresos laborales, respectivamente. Al considerar la ecuación de salarios, los signos y significancia estadística de los coeficientes se mantienen; sin embargo, la magnitud refleja importantes cambios. En particular, las variables escolaridad y experiencia para los economistas presentan menores elasticidades respecto al ingreso laboral; mientras que en la función minceriana propuesta para las demás profesiones se evidencia un efecto marginal mayor tanto en la variable escolaridad como en la experiencia potencial para el periodo de estudio, como se describe en el ejercicio econométrico.

Las diferencias en las tasas de rentabilidad de capital humano en ambos periodos, podrían deberse a un componente no justificado dada la heterogeneidad de capital humano entre los profesionales con distintos reportes salariales, lo que constituye un elemento que sugiere la presencia de un mercado laboral heterogéneo, según la corriente estructuralista. Este constructo empírico refleja que persisten diferencias en el efecto de los determinantes de capital humano sobre los ingresos laborales cuando se consideran de manera separada los grupos de profesionales, pero no ofrece razones suficientes sobre el origen de estas diferencias, solo se infiere que el mercado laboral colombiano no distingue claramente entre profesionales de una u otra área, sino que los graduados compiten entre sí por diferentes oficios, desconociendo la capacidad explicativa de las habilidades y destrezas innatas de los trabajadores, que es comprensible cuando se comparan grupos de profesionales.

Como epílogo se exhibe el ejercicio adicional como prueba empírica de que la cualidad "ser economista" tiene impacto en el logaritmo de los ingresos laborales en Colombia. Luego de hacer la corrección por filtro de selectividad de Heckman, se logró demostrar que el "efecto economista" genera un diferencial positivo de aproximadamente 0.43 y 0.53 en el 
crecimiento de las remuneraciones salariales en el mercado laboral colombiano para los años 2010 y 2016, respectivamente. Ya autores como Jaramillo, Acevedo \& Ortiz (2002) y Forero \& Ramírez (2008) han mencionado que los profesionales del área de Ciencias Económicas y Administrativas que se vinculan a carreras de cualificación de tipo general como Administración de Negocios, Economía y Negocios Internacionales tienen mayor probabilidad de devengar ingresos laborales más altos.

\section{REFERENCIAS BIBLIOGRÁFICAS}

Almendarez M. \& Urciaga, J. \& (2008). Salarios, educación y sus rendimientos privados en la frontera norte de México: Región y sociedad, 20(41), 33-56.

Arrow, K. (1973). Highter Educacion as a Filter. Journal of Public Economics, 2, 193-216.

Becker. (1962). Investment in human capital: A theoretical Analysis. The journal of political economy, 9-49.

Becker. (1964). Human Capital: A Theoretical and Empirical Analysis, with Special Reference to Education. Nueva York: National Bureau of Economic Research.

Ben Porath, Y. (1967). The Production of Human Capital and the Life Cycle of Earnings. The Journal of Political Economy, 75, 352-365.

Brenes Narvaéz, A. (2005). Determinantes del Ingreso laboral en Nicaragua. Documentos de Trabajo del Banco Central de Nicaragua.

Cortés, A., \& Flórez, M. (2016). Diferencias salariales por género en el Departamento de Santander, Colombia. Revista Universidad Industrial de Santander, 271-272.

El-Attar, \& López-Bazo. (2009). Tamaño empresarial y distribución de los salarios. Evidencia para España.

DANE. (2015a). Mercado laboral de la juventud. http://www.dane.gov.co/files/investigaciones/ boletines/ech/juventud/Bol_eje_juventud_mar_may16.pdf

DANE. (2015b). Mercado laboral de la juventud. http://www.dane.gov.co/files/investigaciones/ boletines/ech/juventud/Bol_eje_juventud_mar_may16.pdf

Farné, E. (2000). El mercado de trabajo de los profesionales colombianos. Observatorio del Mercado de trabajo y la seguridad social, 43.

Forero, N. Y., \& Gamboa, L. f. (2007). Cambios en los retornos de la educación en Bogotá entre 1997 y 2003. Revista Universidad de Antioquia.

Forero, N., \& Ramírez, M. (2008). Determinantes de los ingresos laborales de los graduados universitarios en Colombia: Un análisis a partir de la herramienta de seguimiento a graduados. Revista de Economía del Rosario, 87.

Freire, M., \& Mercedes, T. (2010). Las ecuaciones de Mincer y las tasas de rendimiento de la educación en Galicia. Revista Universidad de la Coruña, 2-3. 
Gómez, D., \& Rojas, W. (2014). Análisis de los ingresos de los técnicos y tecnólogos egresados de las ciencias administrativas y afines en Colombia desde la perspectiva del capital humano. TEKCNE, 48-55.

Heckman, J. (1979). Sample selection bias as specification error. Econometrica 47 (1). 61-153.

Iglesias Garrido, J. (2005a). Capital humano y Señalización. Revista de la Universidad Autónoma de Barcelona, 20.

Iglesias Garrido, J. (2005b). Capital humano y Señalización. Revista de la Universidad Autónoma de Barcelona, 20.

Jaramillo, Acevedo \& Ortiz (2002). Impacto social de los programas de pregrado. Centro de Investigaciones económicas y financieras (CIEF). Revista Universidad EAFIT.

Marchionni, Mariana (2001), Determinantes de los ingresos, CEDLAS Universidad Nacional de La Plata.

Martínez Gutiérrez, B. (2012). Efectos de depreciación en capital humano: Evidencia empírica por nivel de cualificación y ramas de actividad en Colombia. Repositorio Universidad del Valle, 6.

McConnell, Brue, \& MacPherson. (2007). Economía laboral. En McConnell, Brue, \& MacPherson, Economía laboral. Madrid: McGraw Hill.

Mincer. (1958). Investment in human capital and personal income distribution. The Journal of political economy. 281-302.

Mincer. (1974). Schooling, experience and earning. NBER Press: New York.

Ortiz Benavides, E., Erazo, I. F., \& Burgos, M. (2015). Educación, empleo e ingresos en el Municipio de Pasto: Un estudio de datos de panel 2008-2010. Tendencias.

Phelps, E. (1972). The Statistical Theory of Racism and Sexism. American Economic Review, 659-661.

Piña. (2006). La formación de capital humano y el mercado laboral Colombiano. Cendex, 13.

Psacharopoulos, G., \& Vélez, E. (1992). Schooling, Ability, and earning in Colombia: 1988. Economic Development and cultural change.

Riascos H., J. C., \& Erazo, I. F. (2010). Situación del mercado laboral de los profesionales en San Juan de Pasto: Un análisis de modelación microeconométrica con datos de corte transversal. Tendencias.

Salas, M. (2003). Determinantes salariales en el mercado de los titulados universitarios. Asociación de economía de la educación, 236.

Sapelli, C. (2003). Ecuaciones de Mincer y las Tasas de Retorno a la Educación en Chile: 1990-1998. Repositorio Pontificia Universidad Católica de Chile.

Schultz, T. (1961). Investment in human capital. The American Economic Review, 1-17.

Schultz, T. (1963). The Economic Value of Education, Columbia University Press. 
Spence, M. (1973). Job Market Signalling. Quarterly Journal of Economics, 87, 355- 374.

Stiglitz, J. (1975). The theory of "screening", education, and the distribution of income, American Economic Review, 65, 283-300.

Tarazona, N., \& Remolina, D. (2017). Efectos de la Tasa de Retorno de la educación en Colombia (20092016). Repositorio Universidad Santo Tomás.

Tenjo, J., Rivero, R., \& Bernat, L. (2005). Evolución de las diferencias salariales en América Latina un intento de interpretación. Documento CEDE.

White, Halbert (1980), A Heteroskedasticity-Consistent Covariance. Michigan: Cengage Learning.

Wooldridge, J. M. (2006). Introducción a la econometría: Un enfoque moderno. Michigan: Cengage Learning. 